\title{
Performance analysis of software defined network using intent monitor and reroute method on ONOS controller
}

\author{
Putri Monika $^{1}$, Ridha Muldina Negara ${ }^{2}$, Danu Dwi Sanjoyo ${ }^{3}$ \\ ${ }^{1,2}$ Adaptive Network Laboratory, School of Electrical Engineering, Telkom University, Indonesia \\ ${ }^{3}$ Cyber Physical System Laboratory, School of Electrical Engineering, Telkom University, Indonesia
}

\section{Article Info \\ Article history: \\ Received Nov 11, 2019 \\ Revised Jan 21, 2020 \\ Accepted Mar 17, 2020}

\section{Keywords:}

Intent monitor and reroute ONOS controller

Quality of service

Software-defined network

Traffic management

\begin{abstract}
Software defined network (SDN) provides high service flexibility to optimize network configuration based on network traffic. Traffic management able to solve traffic density in SDN. However, it will misuse the network bandwidth and links. One variant of the SDN controller, namely open network operating system (ONOS), provides an intent monitor and reroute (IMR) method that can optimize traffic management based on the description of an object in the ONOS application. This method can optimize the network bandwidth and links of SDN. The IMR can monitor the network and reconfigure the network to restore network connectivity by maximizing the use of each link when transmitting data. This study examines the impact of using IMR with a custom topology on ONOS to find the best scenario by performing traffic management on a data plane consisting of switches totaling 8-12 switches. The parameters measured in this study are bandwidth usage and quality of service (QoS). The results obtained in this study are IMR able to optimize the use of each link and maximize bandwidth usage in a network when distributing data and following TIPHON standards.
\end{abstract}

This is an open access article under the CC BY-SA license.

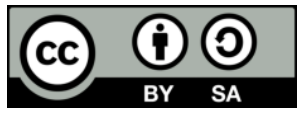

\section{Corresponding Author:}

Ridha Muldina Negara,

Adaptive Network Laboratory, School of Electrical Engineering,

Telkom University,

J1. Telekomunikasi No.1, Bandung, West Java, 40257, Indonesia.

Email: ridhanegara@telkomuniversity.ac.id

\section{INTRODUCTION}

Software defined network (SDN) is an idea put forward by researchers to study networks that are easily programmed by users and simplify the process of managing networks to be more straightforward. Separation of centralized control from data fields becomes the main focus in SDN because it can simplify network management in several ways, one of which is to separate the control plane and the data plane [1-3]. ONOS controller is one of the most deployed controllers in the telecom industry and hence is more demanded research purposes [4]. The SDN controllers have already provided intent-based Northbound interface (NBI): for instance, OpenDaylight [5] and ONOS [6]. Both of them offer network intent composition (NIC) interface and Intent Framework, respectively. In the rest of this paper, we focused on the ONOS controller. The existence of the IMR method provided by ONOS can improve network quality and maximize the use of links on SDN networks. The use of the IMR method on ONOS refers to the problem of network failure in a complex network. The addition of gateway nodes and switches in complex and high availability networks is proportionate to reduce network performance [7, 8]. The IMR service on ONOS enables better traffic management in rerouting the network by using specific purpose and objective utilizing statistical data retrieval from each flow. IMR interacts with both ONOS intent manager and flow rule 
manager to track the processes that occur between intent and flow that correspond to certain information $[9,10]$.

In previous studies, intent monitor and reroute (IMR) was used to see SDN network performance on ONOS controllers by using an additional off-platform application (OPA) that communicates via RestAPI when monitoring and re-routing an SDN network [10]. Whereas, in this study, the implementation of IMR in SDN did not apply additional OPA applications. This research aims to evaluate and analyze the effectiveness of using IMR without these additional applications in managing traffic when maximizing links and bandwidth on the network. The results obtained in this study provide good SDN network quality using the IMR method on the ONOS controller based on bandwidth usage and quality of service (QoS) analysis consisting of delay, jitter, throughput, and packet loss.

\section{PROPOSED METHOD}

\subsection{Software defined network}

SDN is the concept of a network that separates the control plane and the data plane. SDN is flexible and efficient that allows a network to be controlled by software outside the network with physical connectivity. The SDN architecture provides user-friendly functionality so that it can be implemented in the form of software applications that can monitor and modify network status through network operating system services. SDN controller communication to the data fields and application layer can be achieved via (API). The two main APIs in the SDN architecture is the southbound API for communication between controllers and network infrastructure and the northbound API, which defines the interface between network applications and controllers [1]. SDN architecture has a flexible nature, which makes SDN can operate with various types of switches at different protocol layers. SDN controllers and switches can be implemented for Ethernet (Layer 2) switches, Internet routers (Layer 3), transport transfers (Layer 4), and routing [11].

\subsection{ONOS controller}

With the ONOS cloud controller, users can easily create new network applications without the need to change the data plane system. ONOS supports network configuration and control in real-time to eliminates the needs of network routing and switching protocols. ONOS is an open-source distributed under the Apache 2.0 license using a Java-based programming language and uses Apache Karav to bring to life every feature on ONOS. ONOS architecture can be seen in Figure $1[12,13]$. ONOS is an SDN controller with centralized control and has high availability [14].

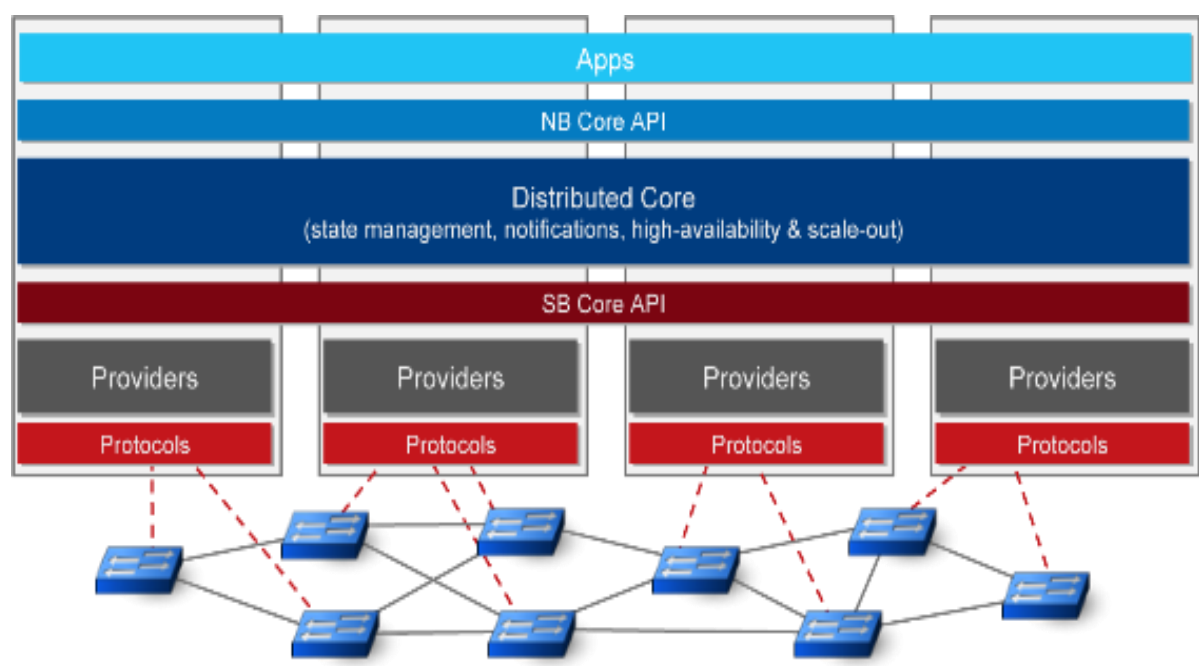

Figure 1. ONOS architecture

\subsection{ONOS intent framework}

Point-to-point intent in the ONOS application works by requesting unidirectional connectivity between 2 hosts on the topology. Intent is a specific subset of traffic with specific values for a packet and is linked to a series of actions in all packages processed. On the ONOS intent framework, users can request that the resulting path passes through a certain number of nodes or bandwidth with a certain amount [12]. 


\subsection{Clustered robust routing}

Clustered robust routing (CRR) allows users to change routing on the network flexibly and dynamically. This algorithm calculates some routing configurations which are going to be applied to the network over the required period. Route configuration is calculated based on traffic irregularities, taking into account the traffic matrix space sub-region [12, 15]. The CRR defines two optimization models, namely:

- Calculation of the routing configuration generating a set of traffic matrices.

- Group a set of traffic matrices that include more details about the optimization model, along with the formulation.

CRR ensues a Python-based application that gathers data from ONOS via the IMR REST API. This algorithm makes it possible to overcome a variety of traffic and failures that can interfere with connectivity. In the IMR service, it is still seen that the intent can effectively recover from network failures $[10,12,15]$. Routing functions to provide a suitable algorithm for service [16].

\subsection{Intent monitor and reroute}

The IMR does filtering for intent data collection statistics. In addition to using applications, users can also monitor using commands on CLI APIs, Rest APIs, and on opensource contributions. The IMR service implementing the ONOS application can optimize routing and make it easy for users to monitor and move routes flexibly. This monitoring is done by taking low-level flow statistics generated by ONOS. IMR currently supports two types of objectives: Point-to-point and link collection. The IMR interacts with ONOS intent manager and flow rule manager to track the destination on the appropriate flow like Figure $2[13,17]$.

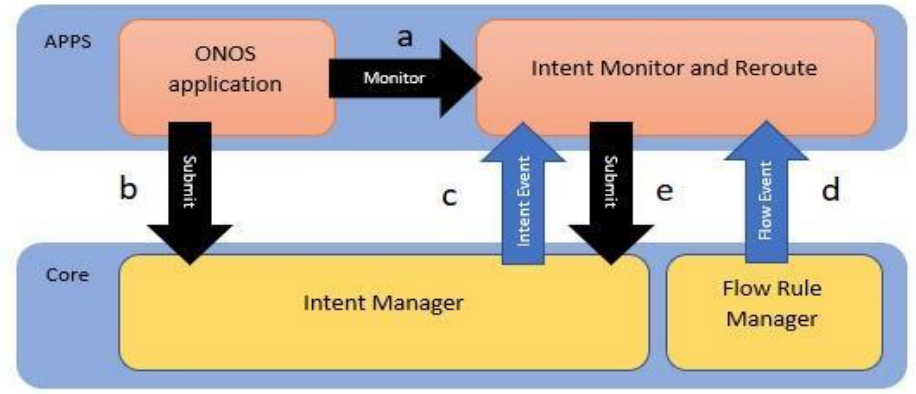

Figure 2. IMR system on ONOS

\subsection{Custom topology}

Custom network topology is a topology that has broad scalability with more links than any other topology. Custom topology is also a random topology and is a combined topology of mesh and linear topology $[18,19]$. Mininet software can develop network infrastructure. Then, a custom network topology can be formed in mininet software [20,21]. Illustration of custom topology in this study can be seen in Figure 3.

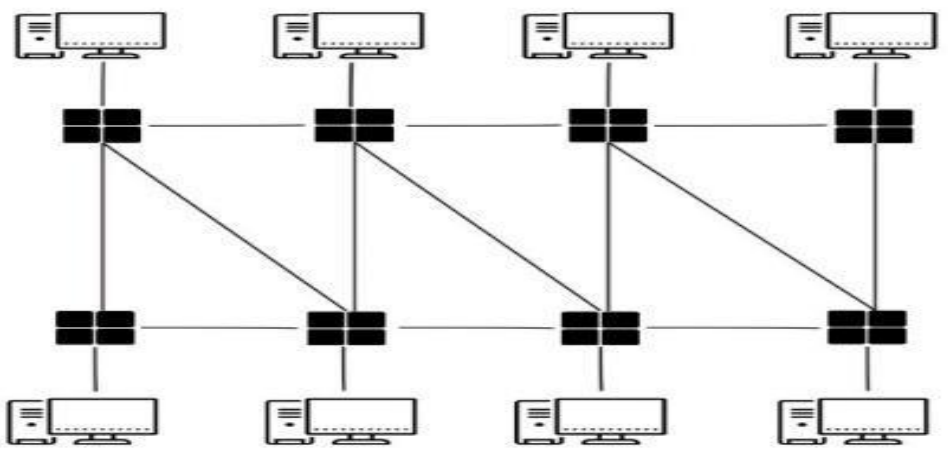

Figure 3. Custom topology illustration 


\subsection{SDN-IP ONOS application}

SDN-IP is an ONOS application that provides connectivity on an SDN network to an IP network using the border gateway protocol (BGP). The SDN network changes routing information through eBGP and provides connectivity to external networks as an autonomous system (AS). The SDN-IP application makes the controller as a passive BGP speaker [12, 22].

Point-to-point intent between external and internal BGP speakers provides connectivity for eBGP peering sessions, while multi-point-to-single-point intent allows connectivity between peering interfaces [12, 23]. In the IMR application, it does not support the multi-point-to-single-point intent, but it supports the point-topoint intent application, as shown in Figure 4.
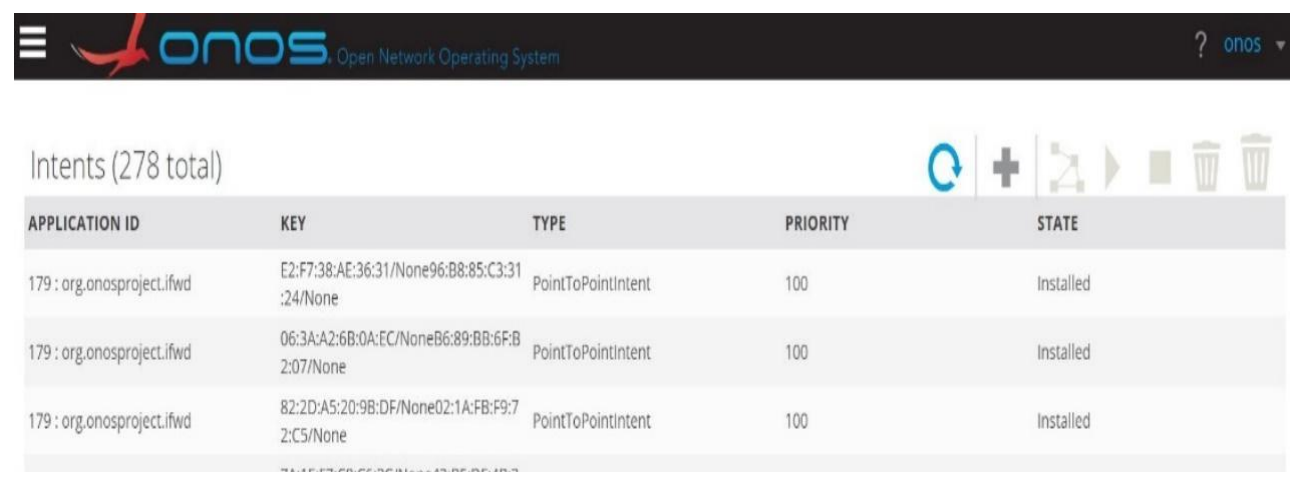

Figure 4. Point-to-point intent on IMR

\subsection{Quality of service}

Quality of service (QoS) is user satisfaction with the quality of a service based on specific parameters. Each service has a different standard of performance value. The test parameters are delay, jitter, throughput, and packet loss [18, 19]. The standardized QoS parameters follows from the TIPHON standard [24]. The service levels of QoS may be priority levels or content-quality levels [25, 26].

\section{RESEARCH METHOD}

This section explains about system design and system implementation in this study. SDN-based network simulations will be tested using IMR on ONOS.

\subsection{System design}

The system design on ONOS as in Figure 5, explains that the system consists of an SDN-based network. Inside the data plane, there is ubuntu version 16.04, which runs a mininet emulator with a network topology, which is a custom topology. In the control plane, there is an ONOS controller that functions as a controller for monitoring SDN networks using IMR.

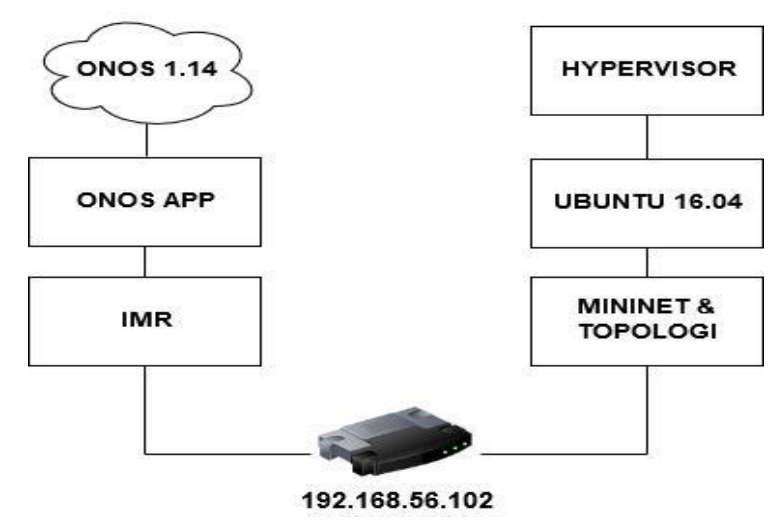

Figure 5. System design 


\subsection{Network topology}

This study uses a custom topology designed on ONOS and uses a different number of switch schemes, namely eight switches, ten switches, and 12 switches. In each network topology used, a maximum bandwidth has been given to each link manually with a value of $10 \mathrm{Mbps}$ each. From the specified scenario, it will be seen the difference in the effectiveness of bandwidth usage and network QoS when using IMR. Each network topology is tested by providing background traffic in stages of $5 \mathrm{Mbps}, 10 \mathrm{Mbps}$, and 15 Mbps. QoS data retrieval is done using D-ITG 2.8.1 when sending data. Data retrieved using D-ITG 2.8.1 are delay, jitter, throughput, and packet loss.

\subsection{System specification}

In the software used in this study, the system specifications are:

- Oracle VM VirtualBox.

- Ubuntu 16.04.

- Mininet as emulator for implementing the testing topology.

- ONOS controller version 1.14. as a network controller.

- Iperf as software tool measuring bandwidth usage and generating background traffic.

- D-ITG 2.8.1 as software tool for acquiring QoS data.

\subsection{Performance scenario}

The testing scenario in this study began with the installation of the ONOS controller version 1.14 on the hypervisor and Ubuntu version 16.04. After the installation is carried out, the system is then simulated using a topology that has been created on the mininet. After the topology appears on the ONOS GUI, the next step is to monitor the network using IMR. After the installation and configuration of the system in the hypervisor, the next step is to turn ONOS on until the CLI from ONOS.

Without the need for modification in IFWD, monitoring and route switching can be done with commands on the CLI localhost "onos".

onos>imr:startmon 161 org.onosproject.ifwd

onos> logout.

Each link in the topology will be used to maximize the sending of data packets, as in Figure 6, that each link on the network topology eight switches all glow green and indicate that the use of IMR on the topology has successfully distributed data packets equally. After the system is declared to work on every topology, the next step is to test to see the parameters of bandwidth usage and QoS in this study.

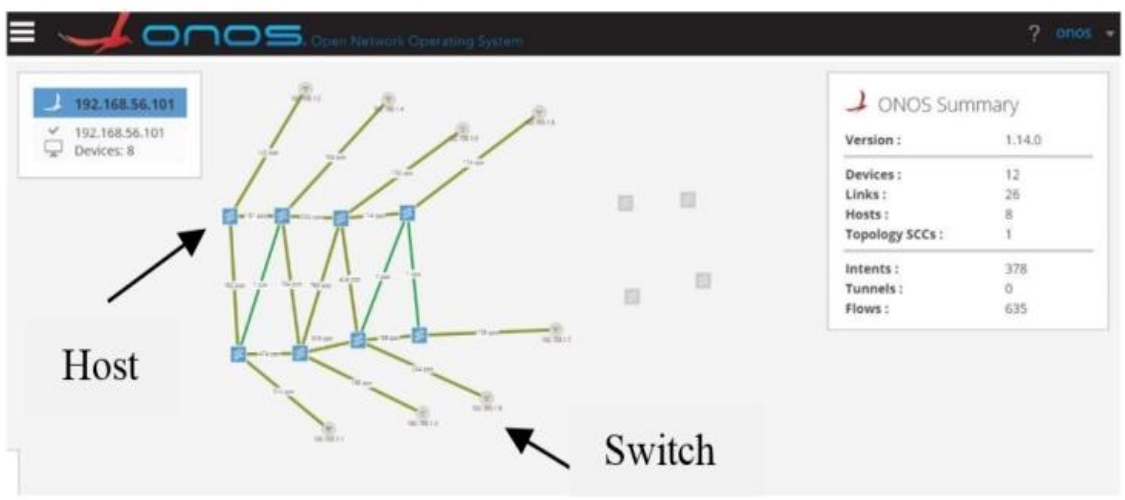

Figure 6. MR was working on topology eight switches

\section{RESULTS AND DISCUSSION}

The analysis was conducted to see the use of the IMR method that affects the performance of the custom topology network. IMR is a new application provided on ONOS and effectively used to monitor a network with excellent performance. Each test is carried out when given background traffic of $5 \mathrm{Mbps}$, $10 \mathrm{Mbps}$, and $15 \mathrm{Mbps}$. The final results and analysis refer to the TIPHON version of the QoS standard.

\subsection{Bandwidth usage test and analysis}

Bandwidth usage is the use of bandwidth on a network that is seen based on the results when the network is given background traffic gradually. The bandwidth test is done to see how much is 
the maximum use of bandwidth on the network, so the quality of the network can be said to be good. If bandwidth usage or bandwidth usage on a network has a value higher than the maximum bandwidth of $10 \mathrm{Mbps}$, then the network leaks when sending data, so it is not feasible to use. From this research, the results obtained show that the more switches on a network, the higher the bandwidth used will be smaller.

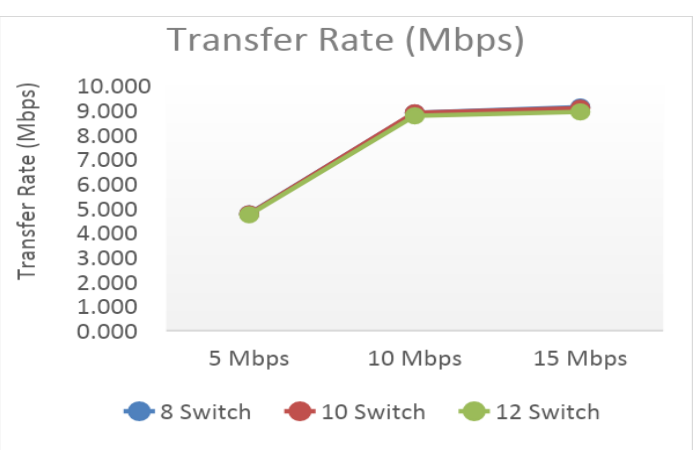

(a)

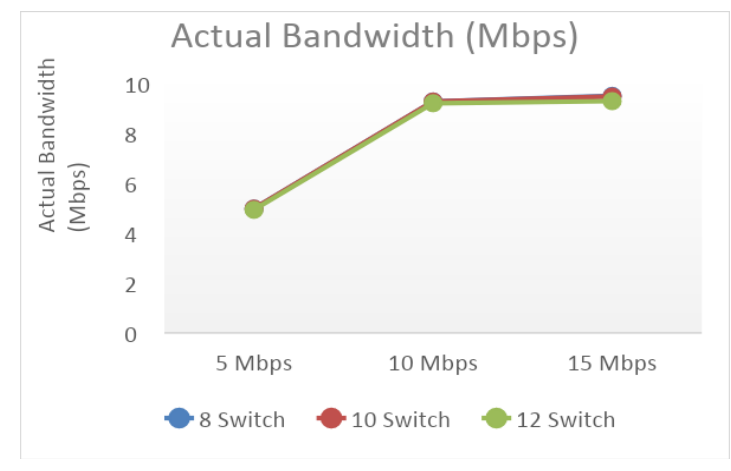

(b)

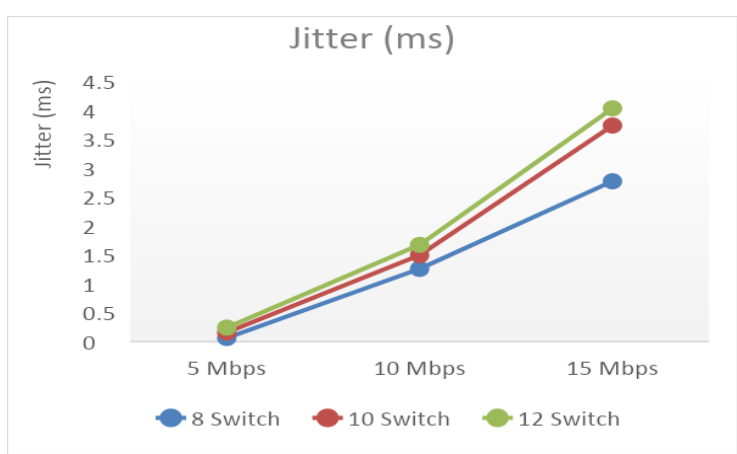

(c)

Figure 7. Bandwidth usage test and analysis graphs of, (a) Transfer rate, (b) Actual bandwidth, and (c) Jitter for each topology

The results prove that sending data packets are distributed to each link equally. When given background traffic of $15 \mathrm{Mbps}$, the transfer rate obtained still does not exceed the maximum bandwidth of 10 Mbps. However, the use of background traffic of $15 \mathrm{Mbps}$ gets a more significant transfer rate than when provided background traffic of $10 \mathrm{Mbps}$, as shown in Figure 7(a). The results of the average actual bandwidth used in each topology show, when topology 12 switches are given background traffic worth 5 Mbps, $10 \mathrm{Mbps}$, and $15 \mathrm{Mbps}$, the actual bandwidth used is smaller than the actual bandwidth used in the topology of 8 switches and ten switches, as shown in Figure 7(b). For the results of jitter in this test, it shows that the value of jitter will increase along with the increasing number of switches used in the network topology, as shown in Figure 7(c).

This test proves that the effectiveness of bandwidth usage by using IMR can be maximized and evenly distributed to each link. Maximum bandwidth on each link becomes the primary reference, which indicates that when given background traffic exceeds the maximum bandwidth on each link, there is no leakage on the network. It can, when given background traffic of $15 \mathrm{Mbps}$, the bandwidth used is still maximized near $10 \mathrm{Mbps}$ and does not exceed $10 \mathrm{Mbps}$, even though it has been given background traffic of 15 Mbps. So, it is proven that bandwidth usage can be maximized when using IMR. The fewer switches are used, the more even distribution of the load and the smaller the use of resources and the risk of data congestion.

\subsection{QoS test and analysis}

QoS testing is done to see the performance or quality of an SDN network on ONOS using IMR. This test is carried out using 8 Switch, 10 Switch, and 12 Switch topology networks when running IMR and 
then see the network performance on the topology using D-ITG 2.8.1 to see the QoS results. After IMR has worked on each network topology, the next step is to test each topology by sending UDP packets from the client to the server.

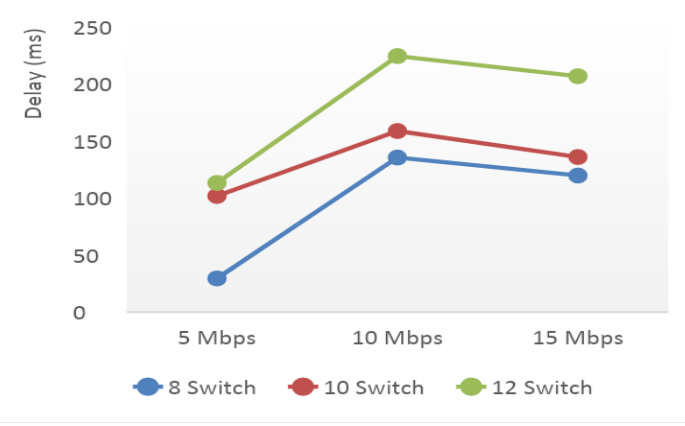

(a)

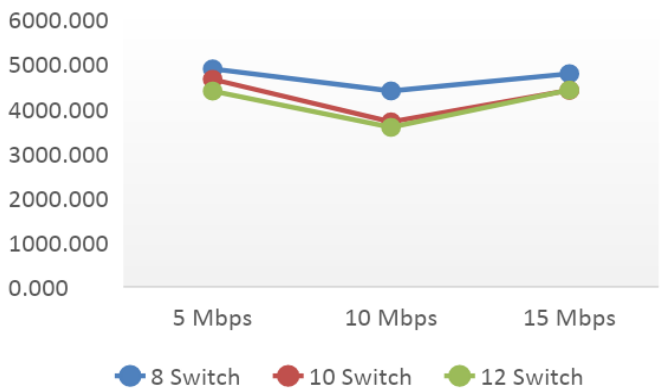

(c)

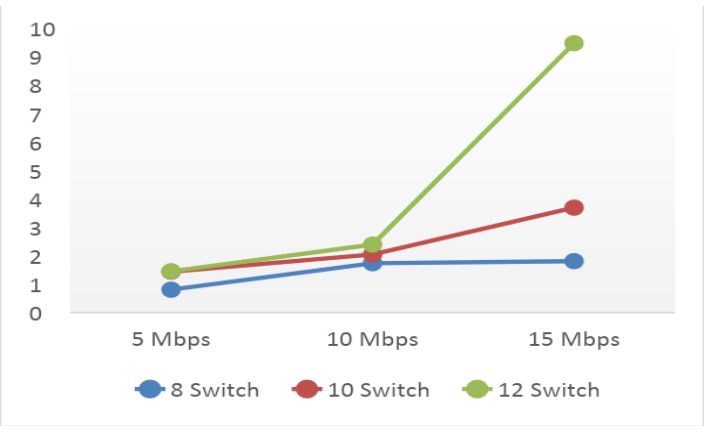

(b)

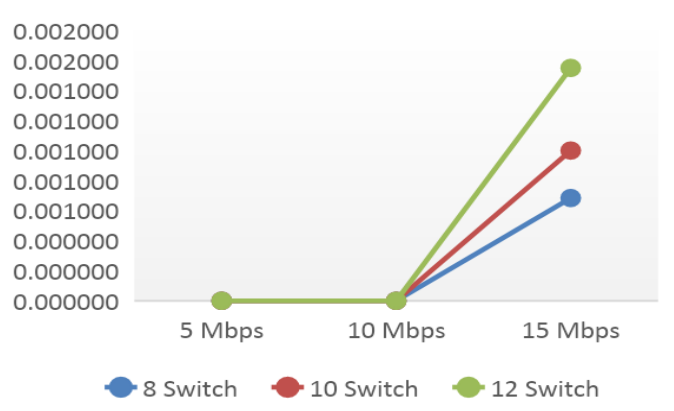

(d)

Figure 8. QoS test and analysis graphs of (a) Delay, (b) Jitter, (c) Throughput, and (d) Packet loss of each topology

The delay value is the packet delay time when it is transmitted from the starting point to the destination point. The results contained in this test indicate that the delay when given background traffic of $15 \mathrm{Mbps}$ is the highest value on each network topology used. The higher the background traffic provided, the higher the delay value, as shown in Figure 8(a). The value of jitter is a variation of delay whose results will be directly proportional to the delay. By this result, it can be seen that an increase in jitter value according to that is influenced by an increasing number of Switches and background traffic, as shown in Figure 8(b).

Throughput is the amount of value that shows the actual bit rate of data information from the bit rate on the telecommunications network. The higher the throughput, the better the quality of the network. The throughput value can be seen in the results shown in Figure 8(c). The average throughput value in this test shows that the results are still following the TIPHON version of the QoS standard. In testing the system simulation using IMR, there is no packet loss. Because every network topology is given a load that does not exceed the maximum bandwidth on each link that is $10 \mathrm{Mbps}$, however, when given a data load or background traffic of $15 \mathrm{Mbps}$, get a packet loss value that varies depending on the number of switches listed in Figure 8(d).

The results of testing in terms of QoS using D-ITG 2.8.1 in this simulation show that the quality of the SDN network when using IMR has good quality. The average delay value in this simulation is excellent, with delays ranging from $150 \mathrm{~ms}$ to $300 \mathrm{~ms}$ according to the TIPHON standard. The value of throughput also falls into the excellent category. The condition is occurring because the average throughput shows the amount of throughput $>1,200 \mathrm{Kbps}$. Similarly, the results contained in the packet loss value has a perfect result with a significant $0 \%$ and does not exceed $3 \%$. 


\section{CONCLUSION}

Thus from the results of system testing and analysis on the software network defined network using the IMR method do yield good network result and performance, and it is feasible to use to support users in using SDN-based networks on ONOS.

This research shows that IMR is proven to be able to optimize the use of links and maximize bandwidth usage in a network when distributing data. The effectiveness of bandwidth usage on each topology gives good results because bandwidth usage does not exceed the maximum bandwidth that has been manually set on each link in each topology of $10 \mathrm{Mbps}$. Increasing the number of switches can affect the distribution of bandwidth evenly becomes smaller. Therefore, in this simulation, it can be said that the number of switches affects each parameter tested. However, the number of switches does not affect the quality of SDN tested using IMR. The results can show the optimal network quality for each network topology in this study

\section{REFERENCES}

[1] D. Kreutz, et al., "Software-defined networking: A comprehensive survey," arXiv preprint arXiv:1406.0440, 2014.

[2] T. A. Limoncelli, "Openflow: a radical new idea in networking," Queue, vol. 10, no. 6, p. 40, 2012.

[3] R. Dhaya, et al., "Software defined networking: Viewpoint from IP networking, PROS nd CONS and exploration thoughts," in Conference of the 2017 International Conference on Intelligent Computing and Control Systems, ICICCS 2017, June 2017.

[4] R. K. Arbettu, et al., "Security analysis of OpenDaylight, ONOS, Rosemary and Ryu SDN controllers," in 2016 17th International Telecommunications Network Strategy and Planning Symposium (Networks), Sep 2016.

[5] J. Medved, et al., "OpenDaylight: Towards a Model-Driven SDN Controller architecture," in Proceeding of IEEE 15th International Symposium on a World of Wireless, Mobile and Multimedia Networks 2014, 2014.

[6] P. Berde, et al., "ONOS: Towards an open, distributed SDN OS," in HotSDN 2014-Proceedings of the ACM SIGCOMM 2014 Workshop on Hot Topics in Software Defined Networking, pp. 1-6, 2014.

[7] R. Tulloh, et al., "Load distribution analysis on bipartite topology using floodlight controller," Journal of Theoretical and Applied Information Technology, vol. 96, no. 5, pp. 1238-1252, 2018

[8] A. R. D. Nugraha, et al., "High Availability Performance on OpenDayLight SDN Controller Platform (OSCP) Clustering and OpenDayLight with Heartbeat-Distributed Replicated Block Device (DRBD)," Jurnal Infotel, vol. 10, no. 3, pp. 149-156, 2018.

[9] D. Sanvito, D. Moro, M. Gullì, I. Filippini, A. Capone and A. Campanella, "Enabling external routing logic in ONOS with Intent Monitor and Reroute service," 2018 4th IEEE Conference on Network Softwarization and Workshops (NetSoft), Montreal, QC, pp. 332-334, 2018.

[10] D. Sanvito, D. Moro, M. Gullì, I. Filippini, A. Capone and A. Campanella, "ONOS Intent Monitor and Reroute service: enabling plug\&play routing logic," 2018 4th IEEE Conference on Network Softwarization and Workshops (NetSoft), Montreal, QC, pp. 272-276, 2018.

[11] M. S. Olimjonovich, "Software Defined Networking: Management of network resources and data flow," 2016 International Conference on Information Science and Communications Technologies (ICISCT), pp. 1-3, 2016.

[12] A. Rajaratnam, et al., "Software defined networks: Comparative analysis of topologies with ONOS," in Proceedings of the 2017 International Conference on Wireless Communications, Signal Processing and Networking, WiSPNET 2017, vol. 2018-Jan, pp. 1377-1381, 2018.

[13] D. T. R. ETSI, "TIPHON-05001, Telecommunications and Internet protocol harmonization over networks (TIPHON)," General Aspects of quality of service (QoS), 1998.

[14] A. Attari, et al., "Analisis Performansi High Availability Jaringan pada Virtual Private LAN Service Legasi dan Berbasis Software Defined Network - Analysis of High Availability Network Performance on Legacy Virtual Private LAN Services and Software Defined Network Based," Prosiding SENIATI, vol. 5, no. 3, pp. 99-104, 2019.

[15] D. Sanvito, et al., "Adaptive Robust Traffic Engineering in Software Defined Networks," in 2018 IFIP Networking Conference IFIP Networking and Workshops, IFIP Networking 2018 - Proceedings, pp. 145-153, 2018.

.[16] H. A. Naqvi, et al., "Enabling multipath routing for unicast traffic in Ethernet network," in 2015 3rd International Conference on Information and Communication Technology (ICoICT), pp. 245-250, 2015.

[17] X. Foukas, et al., "Software Defined Networking Concepts," in Software Defined Mobile Networks (SDMN), Chichester, UK: John Wiley \& Sons, Ltd, pp. 21-44, 2015.

[18] W. Kim, J. Li, J. W. Hong and Y. Suh, "OFMon: OpenFlow monitoring system in ONOS controllers," 2016 IEEE NetSoft Conference and Workshops (NetSoft), pp. 397-402, 2016.

[19] M. Karakus, and A. Durresi, "Quality of Service (QoS) in Software Defined Networking (SDN): A survey," Journal of Network and Computer Applications, vol. 80, pp. 200-218, Feb 2017.

[20] S. Veena, et al., "A Framework for Implementing Realistic Custom Network Topology in Mininet," International Journal of Science and Research (IJSR), vol. 3, no. 7, pp. 1316-1323, Jul 2014.

[21] F. Keti and S. Askar, "Emulation of Software Defined Networks Using Mininet in Different Simulation Environments," 2015 6th International Conference on Intelligent Systems, Modelling and Simulation, pp. 205-210, 2015.

[22] O. P. Jaya, et al., "Performansi High Availability pada Software Defined Network-Internet Protocol untuk Topologi Jaringan Inti," Prosiding SENIATI, vol. 5, no. 3, pp. 209-214, 2019. 
[23] P. L. Ventre et al., "SDN-Based IP and Layer 2 Services with an Open Networking Operating System in the GÉANT Service Provider Network," in IEEE Communications Magazine, vol. 55, no. 4, pp. 71-79, April 2017.

[24] C. Morin, et al., "On demand QoS with a SDN traffic engineering management (STEM) module," in 2017 13th International Conference on Network and Service Management, CNSM 2017, vol. 2018-Jan, pp. 1-6, 2017.

[25] J. Karaoguz, et al., "Quality-of-service (QoS)-based association with a new network using background network scanning." Google Patents, 2015.

[26] P. Tapia, and M. Shah, "Quality of service adjustments to improve network utilization." Google Patents, 2017.

\section{BIOGRAPHIES OF AUTHORS}
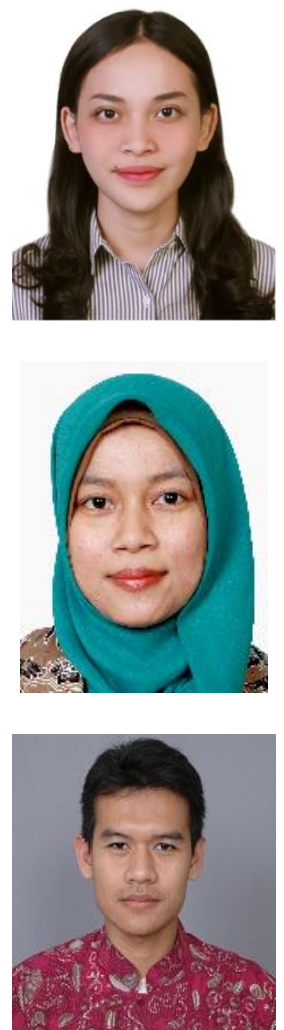

Putri Monika received her BSc degrees in Telecommunication Engineering, from the Telkom University, Indonesia in 2019, respectively. Her interests include Software Defined Networks, and Virtualization Network Engineering.

Ridha Muldina Negara received her Bachelor of Engineering (S.T.) and Master of Engineering (M.T.) degrees in Telecommunication Engineering, from Telkom University, Indonesia in 2009 and 2013, respectively. Since 2010, she has been a Lecturer at School of Electrical Engineering, Telkom University. Her interests include Software Defined Networks, Cyber Security, Telecommunication Systems and Computer Engineering

Danu Dwi Sanjoyo received his Bachelor of Engineering (S.T.) and Master of Engineering (M.T.) degrees in telecommunication engineering from Telkom University, Indonesia in 2010 and 2013, respectively. He is currently pursuing Ph.D. degree in information security with the Division of Electrical Engineering and Computer Science, Graduate School of Natural Science and Technology, Kanazawa University, Japan. Since 2014, he has been a Lecturer at School of Electrical Engineering, Telkom University. His research interests include information security, data privacy, internet of thing, software defined networks, network virtualization, and also compressive sensing. 